Article

\title{
Experimental study on the heat transfer performance of pump- assisted capillary phase-change loop
}

\author{
Xiaoping Yang ${ }^{1}$, Gaoxiang Wang ${ }^{1}$, Cancan Zhang ${ }^{2}$, Jie Liu' ${ }^{1}$ and Jinjia Wei ${ }^{1,3^{*}}$ \\ 1 College of Chemical Engineering and Technology, Xi'an Jiaotong University, Xi 'an, Shaanxi, China; yx- \\ ping@xjtu.edu.cn; 1458241625@qq.com \\ 1 Key Laboratory of Enhanced Heat Transfer and Energy Conservation, Ministry of Education of China, Col- \\ lege of Environmental and Energy Engineering, Beijing University of Technology, Beijing, China; \\ zcc@bjut.edu.cn \\ 3 State Key Laboratory of Multiphase Flow in Power Engineering, Xi'an Jiaotong University, Xi 'an, Shaanxi, \\ China; jjwei@xjtu.edu.cn \\ * Correspondence: jjwei@xjtu.edu.cn; Tel. +86-029-82664375.
}

Featured Application: The pump-assisted capillary phase-change loop overcomes the two-phase flow instability of traditional boiling heat dissipation technologies. Thus it is prospective in heat dissipation of electronics devices and batteries on ground and in space, such as datacenter cooling, thermal management of batteries or spacecraft.

\begin{abstract}
To overcome the two-phase flow instability of traditional boiling heat dissipation technologies, a porous wick was used for liquid-vapor isolation, thus realizing efficient and stable boiling heat dissipation. A pump-assisted capillary phase-change loop with methanol as working medium was established to study the effect of liquid-vapor pressure difference and heating power on its start-up and steady-state characteristics. The results indicated that the evaporator undergoes four heat transfer modes including flooded, partial flooded, thin film evaporation and overheating. The thin film evaporation mode was the most efficient one with the shortest start-up period. Besides, the heat transfer modes were determined by liquid-vapor pressure difference and power. The heat transfer coefficient could be significantly improved and the thermal resistance could be reduced by increasing liquid-vapor pressure difference as long as it did not exceed $8 \mathrm{kPa}$. However, when the liquid-vapor pressure difference exceeded $8 \mathrm{kPa}$, its influence on the heat transfer coefficient weakened. In addition, a two-dimensional heat transfer mode distribution diagram considering both liquid-vapor pressure difference and power was drawn through a great number of experiments. During engineering application, the liquid-vapor pressure difference can be controlled to maintain efficient thin film evaporation in order to achieve the optimum heat dissipation effect.
\end{abstract}

Keywords: liquid cooling; phase-change loop; pressure difference; heat transfer enhancement

\section{Introduction}

Since the heat transfer coefficient of phase-change heat dissipation is $1 \sim 2$ orders of magnitude higher than single-phase convection heat dissipation. Hence, phase-change heat dissipation technology can be adopted to greatly save pumping power and also ensure more compact heat dissipation structure [1,2]. For this reason, the phase-change heat dissipation technology has attracted extensive attention in the field of thermal management of electronic devices under the conditions of normal gravity on the ground and microgravity in the space. Due to its high efficiency and compactness, the phase-change heat dissipation technology has been applied to the cooling of electronic devices on the spacecraft (such as satellites, spaceships and space stations) earlier such as passive loop heat pipe (LHP) [3] and capillary pumped loop (CPL) [4] and active pump-driven two-phase loop [5]. Phase-change heat dissipation displays its high efficiency under the condition of normal gravity on the ground, enabling it to be widely applied in the fields of high heat 
flux chip cooling and thermal management of battery, with the pump-driven micro/mini channel cooling plate as the main form $[6,7]$. However, the phase-change cooling system may become unstable in its pressure, temperature and flow during operation, which may not only reduce the critical heat flux but also cause such problems as vibration, noise and local hot spots $[8,9]$. Therefore, it has become a focus of research how to overcome twophase flow instability in the phase-change cooling system.

For years, many scholars have carried out researches on the mechanism of two-phase flow instability in phase-change cooling system from both macro-scale $[10,11]$ and microscale channels[12-15]. Ding et al. [10] carried out an experimental study on the pressure pulsation caused by flow boiling in the horizontal pipe with a diameter of $10.9 \mathrm{~mm}$ earlier and found three types of pulsation i.e. pressure drop type, density wave type and thermal type. According to the dynamic pressure test results, the pressure pulsation amplitude of pressure drop type instability may reach $160 \mathrm{kPa}$ with frequency less $3 \mathrm{~Hz}$, which may easily cause pipeline resonance. With significant advantages such as strong heat transfer capacity, compact structure and easy integration, the micro-channel flow boiling is very promising in the field of thermal management of high heat flux electronic devices [16, 17]. However, as a matter of fact, the application of two-phase flow is limited due to its instability. Wu and Cheng [12] carried out a synchronous visualization research. They found that the flow patterns of micro-channel flow boiling are closely related to temperature, pressure and flow pulsation. In particular, alternate flow of liquid/vapor/liquid-vapor under high heat flux and low mass flux may cause strong pulsation, with pressure fluctuation amplitude up to $44 \mathrm{kPa}$ and temperature fluctuation to $229^{\circ} \mathrm{C}$. It shows that there are many types of two-phase flow instability in the phase-change cooling system, which are closely related to the transition in flow patterns. Flow pattern is a special physical phenomenon of vapor phase dispersion in the liquid phase. The compressibility of liquidvapor two-phase flow and evolution of vapor volume fraction may cause the fluctuations in both upstream and downstream flow field parameters (pressure, temperature and flow rate).

The surface micro/nano structure can be used in the micro-channel to increase the nucleate site density, promote the nucleation of bubbles at low wall superheat, and restrain boiling delay, thus not only eliminating the two-phase instability but also enhancing boiling heat transfer [18-20]. Some scholars have put forward some methods for active suppression of micro-channel flow boiling instability, such as increasing the inlet liquid pressure to prevent backflow [21], using subcooled liquid jet to prevent bubbles from growing $[22,23]$, etc. However, the micro/nano structure is very complicated to prepare, and cannot be used for active control of instability and heat transfer process. Active regulating methods may cause large flow pressure drop and more extra pump work.

The root cause of two-phase flow instability in the phase-change cooling system is various liquid-vapor two-phase flow phenomena in the phase-change process. In this study, an idea of isolating vapor phase from liquid phase based on the above analysis is proposed. The specific method is as follows: a porous capillary wick is set between the evaporation surface and the liquid channel, so that liquid can be supplied to the evaporation surface through the liquid permeability of capillary wick. The gas produced by phasechange can be isolated through the vapor isolation characteristics of the capillary wick, which prevents the formation of liquid-vapor two-phase flow, thus solving the two-phase instability of phase-change cooling system. By establishing a pump-assisted capillary phase-change loop system with methanol as working medium, this paper studies the effect of liquid-vapor pressure difference on both sides of the capillary wick on the start-up characteristics and steady-state heat transfer characteristics. The proposed system is found to be good overcoming two-phase flow instability. Meanwhile, the heat transfer mode of evaporator can be effectively controlled by controlling the liquid-vapor pressure difference to enable the evaporator to form efficient thin film evaporation heat transfer and significantly enhance heat transfer. 


\section{Experimental system}

\subsection{Experimental apparatus}

Figure 1 presents the system sketch of pump-assisted capillary phase-change loop. The phase-change loop consists of evaporator, liquid storage tank, diaphragm pump, vapor/liquid pipelines, simulated heating system, valve, temperature/pressure sensors and data acquisition system, etc. The working medium methanol was stored in the liquid storage tank, with its temperature controlled by an industrial chiller. The diaphragm pump delivered the liquid working medium through the liquid line to the upper compensation chamber of the evaporator. A part of the liquid entered the baseplate of evaporator for heat dissipation through the capillary wick while the remaining liquid flowed out of the other end of the compensation chamber to the liquid storage tank. After entering the baseplate of evaporator, the liquid absorbed heat and phase-change occurred, and then flowed out of the gas pipeline to the liquid storage tank for condensation. Pressure measuring points were set at the outlets of both compensation chamber and vapor collector to monitor both the pressure of subcooled liquid PL and the pressure of hot fluid PV. Meanwhile, high precision regulating valves were installed at the liquid lines at both inlet and outlet of the compensation chamber to regulate the liquid pressure of the compensation chamber, in order to control the liquid-vapor pressure difference between both sides of the capillary wick to be $\Delta P_{\mathrm{LV}}=P_{\mathrm{L}-} P_{\mathrm{V}}$. The thermophysical properties of the working medium methanol are shown in Table 1.

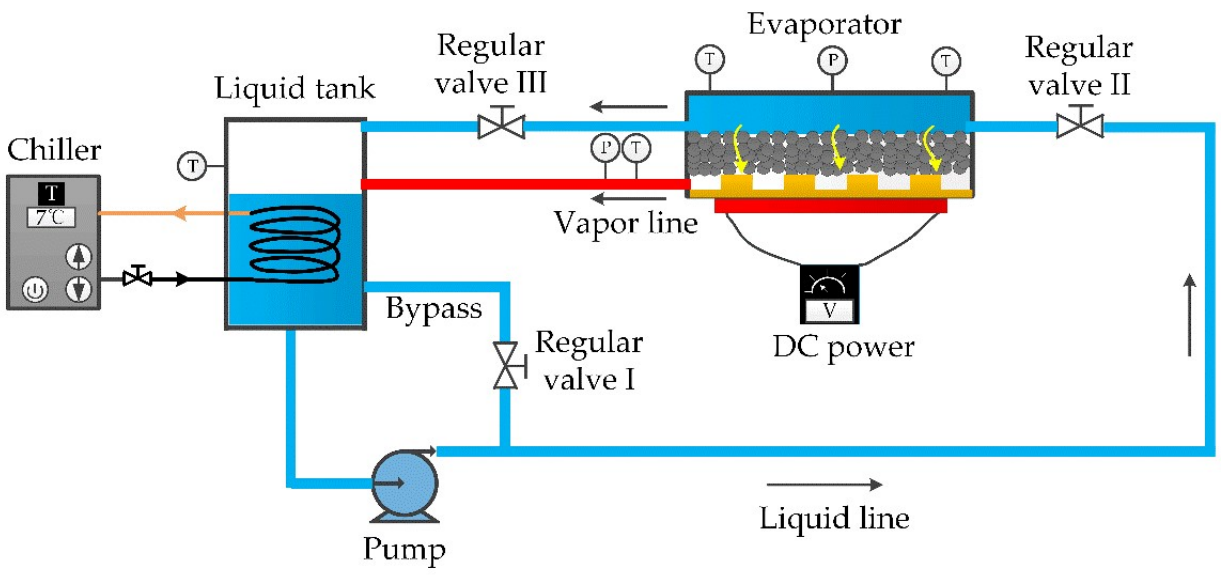

Figure 1. Schematic diagram of the pump-assisted capillary phase-change loop.

Table 1. Thermophysical properties of methanol at room temperature and pressure

\begin{tabular}{ccc}
\hline Parameters & Unit & Value \\
\hline Boiling point & ${ }^{\circ} \mathrm{C}$ & 64.7 \\
Freezing & ${ }^{\circ} \mathrm{C}$ & -97.8 \\
Kinematic viscosity & $\mathrm{mPa} \cdot \mathrm{s}$ & 0.59 \\
Latent heat & $\mathrm{kJ} / \mathrm{kg}$ & 1109 \\
Surface tension & $\mathrm{mN} / \mathrm{m}$ & 22.6 \\
Specific heat capacity & $\mathrm{kJ} / \mathrm{kg} \cdot \mathrm{K}$ & 2.51 \\
Density & $\mathrm{kg} / \mathrm{m}^{3}$ & 792 \\
\hline
\end{tabular}

\subsection{Evaporator}


The evaporator is a core part of pump-assisted capillary phase-change loop, including upper cover plate, capillary wick, copper baseplate, heating plate and support plate from top to bottom as shown in Figure 2. Compensation chamber, liquid inlet/outlet channels and vapor collector were set on the upper baseplate while pressure measuring points were set on the sides of compensation chamber to monitor fluid pressure. The capillary wick was located between the upper cover plate and copper baseplate and also embedded directly below the compensation chamber. The copper baseplate was close to the capillary wick, with rubber ring used to achieve fluid seal. The heating plate was attached to the copper baseplate through thermally conductive silicone grease. The main dimensions of the evaporator are shown in Table 2.

The capillary wick is rectangular with a size of $23 \times 23 \times 5 \mathrm{~mm}^{3}$. It is made by sintering the spherical nickel powder particles with particle size of $20 \mu \mathrm{m}$ by sintering process of cold press sintering under the sintering temperature of $840{ }^{\circ} \mathrm{C}$. In order to improve the porosity of capillary wick, $\mathrm{Na}_{2} \mathrm{CO}_{3}$ particles with mass fraction of $15 \%$ were added as pore former to the nickel powder particles. After sintering, the capillary wick can be obtained through ultrasonic cleaning with deionized water.

The copper baseplate was made of red copper. Square grooves were made on the upper surface by machining for vapor flow. There were totally 11 square grooves with a size of $1 \times 1 \times 23 \mathrm{~mm}^{3}$. A vapor collecting region was set at the end of the grooves and connected with vapor collector on the upper cover plate to form a complete vapor flow path. The back of copper baseplate was connected to the heating plate through thermally conductive silicone grease.

The heating plate is phosphorus doped silicon plate with resistance of about $50 \Omega$ and size of $20 \times 20 \times 0.5 \mathrm{~mm}^{3}$. The electrodes were prepared at both ends of the heating plate by ultrasonic welding and connected to a DC power supply to generate joule heat. The voltage at both ends of the electrode can be controlled to limit the heating power.

See Table 3 for the range of experimental conditions in this paper. The liquid inlet temperature of the compensation chamber was maintained at $10{ }^{\circ} \mathrm{C}$. The heating power was 10 81 W while the liquid-vapor pressure difference was $0 \sim 22 \mathrm{kPa}$.

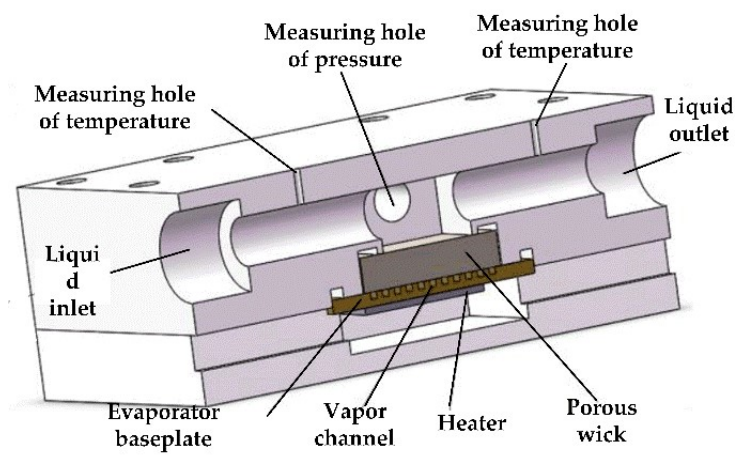

(a)

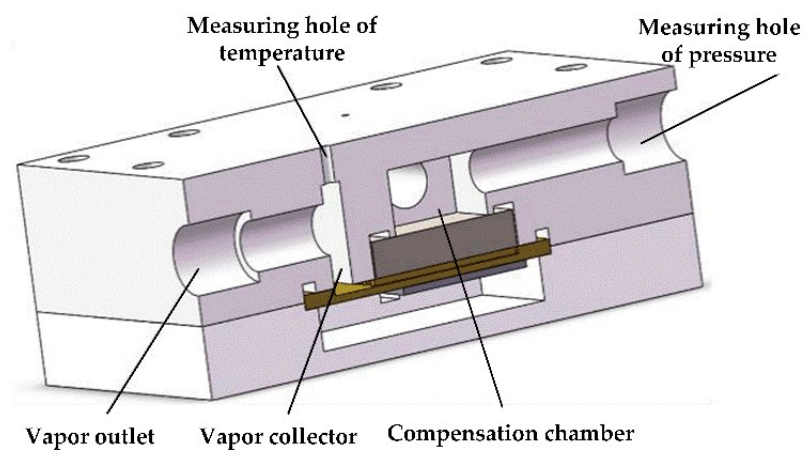

(b)

Figure 2. 3-dimensional diagram of the evaporator, a) Front view of cross-section drawn; b) Side view of cross-section drawn.

Table 2. Main dimensions and test conditions in the experiments

\begin{tabular}{lccc}
\hline Components & Parts & Material & Dimensions \\
\hline & & & Overall size: $36 \times 30 \times 2 \mathrm{~mm}^{3}$ \\
Baseplate & Copper & Vapor channel size: $1 \times 1 \times 23 \mathrm{~mm}^{3}$ \\
& & Vapor channel number: 11 \\
& Wick & Sintered nickel & Overall size: $23 \times 23 \times 5 \mathrm{~mm}^{3}$ \\
& & powder & Particle size: $20 \mu \mathrm{m}$ \\
\hline
\end{tabular}


Pore-forming agent content: $15 \% \mathrm{wt}$

Porosity: $33 \%$

Permeability: 2.99E-11

Average pore size: $2 \mu \mathrm{m}$

Compensation

chamber

Polycarbonate

$60 \times 20 \times 12 \mathrm{~mm}^{3}$ (inner)

\begin{tabular}{|c|c|c|c|}
\hline Heater & Substrate & Silicon & $\begin{array}{l}\text { Active heating area: } 20 \times 20 \mathrm{~mm}^{2} \\
\text { Resistance: } 50 \Omega\end{array}$ \\
\hline
\end{tabular}

Table 3. Test conditions

\begin{tabular}{ccc}
\hline Parameters & Unit & Value \\
\hline Inlet liquid temperature, $T_{\mathrm{cc}}$ & ${ }^{\circ} \mathrm{C}$ & 10 \\
Heat sink temperature, $T_{\text {sink }}$ & ${ }^{\circ} \mathrm{C}$ & 5 \\
Heating power, $Q$ & $\mathrm{~W}$ & $10 \sim 80$ \\
Heat flux, $q$ & $\mathrm{~W} / \mathrm{cm}^{2}$ & $2.5 \sim 20$ \\
Liquid-vapor pressure difference, $\Delta P_{\mathrm{LV}}$ & $\mathrm{kPa}$ & $0 \sim 22$ \\
\hline
\end{tabular}

\subsection{Measurement and uncertainty}

Pressure measurement was carried out by using an absolute pressure transmitter with a range of $0 \sim 600 \mathrm{kPa}$ and accuracy of $0.25 \mathrm{FS} \%$. The pressure measuring points were respectively set on the side wall of compensation chamber and the vapor pipeline near the vapor collector. Temperature measurement was carried out by using a T-type thermocouple, with a range of $-200 \sim 350{ }^{\circ} \mathrm{C}$ and accuracy of $\pm 0.5{ }^{\circ} \mathrm{C}$. There were 6 temperature measuring points at the inlet/outlet of compensation chamber, upper surface of copper baseplate, outlet of vapor collector, liquid storage tank and simulated heat source surface respectively. Digital display DC power supply was used monitor both current and voltage of simulated heat source, with measurement accuracy of current and voltage of $0.01 \mathrm{~A}$ and $0.1 \mathrm{~V}$ respectively. Under current working conditions, the uncertainties of pressure, liquid-vapor pressure difference, temperature, current, voltage and power measurement was $0.9 \%, 1.3 \%, 3.0 \%, 3.0 \%, 0.4 \%$ and $3.1 \%$ respectively.

\section{Results}

\subsection{Start-up characteristics}

The start-up characteristics of pump-assisted capillary phase-change loop under liquid-vapor pressure differences $\Delta P_{\mathrm{Lv}}=0 \mathrm{kPa}, 2 \mathrm{kPa}, 4 \mathrm{kPa}, 6 \mathrm{kPa}, 8 \mathrm{kPa}$ and $16 \mathrm{kPa}$ are shown in Figures 3(a) (f) respectively. By comparison, it can be found that when $\Delta P_{\mathrm{LV}}=0$ $\mathrm{kPa}$ (Figure 3a), the start-up characteristics of phase-change loop are different from those at $\Delta P_{\mathrm{LV}}>0 \mathrm{kPa}$. With the increase of heating power, the outlet temperature $T_{\mathrm{v}}$ of vapor collector increased first, then maintained at $65{ }^{\circ} \mathrm{C}$ (saturation state) and finally increased to about $80^{\circ} \mathrm{C}$ (overheating state). The heat transfer modes of copper baseplate can be divided into four types i.e. flooded, partial flooded, thin film evaporation and overheating according to the change in $T_{\mathrm{v}}$. From the perspective of stable temperature (horizontal section), the heat transfer process was dominated by single-phase convection under low heating power, and the heat transfer efficiency was low with relatively higher temperature of copper baseplate $T_{\text {eva. }}$. At that time, the heat transfer modes were flooded and partial flooded. With the increase of heating power, the heat transfer mode changed to efficient thin film evaporation. With the increase of heating power, the increase magnitude of $T_{\text {eva }}$ decreased. When the heating power exceeded $38 \mathrm{~W}$, the liquid could not completely infiltrate the copper baseplate, and part of the vapor channel began to be exposed, the vapor 
at the outlet of the vapor collector became overheated. At that time, the heat transfer mode was overheating. As for the time required to reach stable temperature (rise section), it took more than 500s under flooded and partial flooded but less than 100s under thin film evaporation.

When the liquid-vapor pressure difference increased to above $2 \mathrm{kPa}$, the liquid replenishment capacity was enhanced, the overheating mode was avoided. Meanwhile, with the increase of liquid-vapor pressure difference, the copper baseplate temperature $T_{\text {eva }}$ decreased under the same heating power. Besides, the difference between $T$ eva and the vapor collector temperature $T_{\mathrm{v}}$ under high power decreased, indicating that the liquidvapor pressure difference could enhance the heat dissipation capacity of phase-change loop.

Besides, according to the start-up characteristics of phase-change loop under various liquid-vapor pressure differences, the copper baseplate temperature $T_{\text {eva }}$ was relatively stable under both low power and high power, without temperature fluctuation or temperature overshoot, indicating that phase-change loop could eliminate the two-phase flow instability.

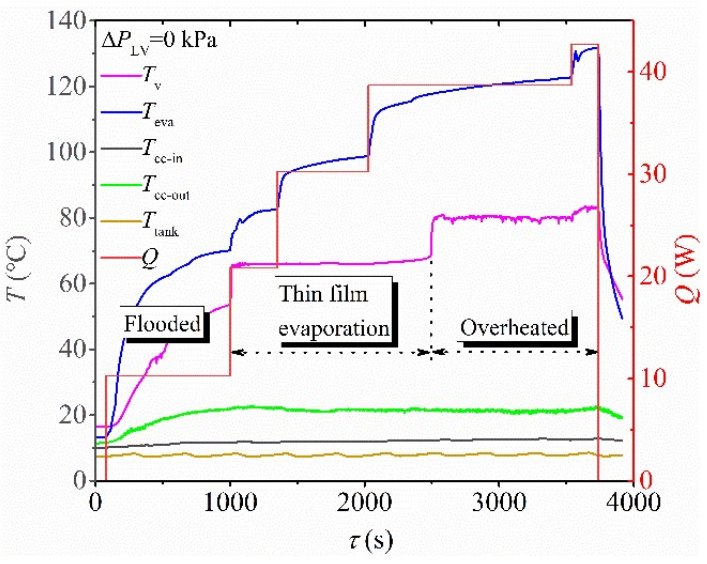

(a)

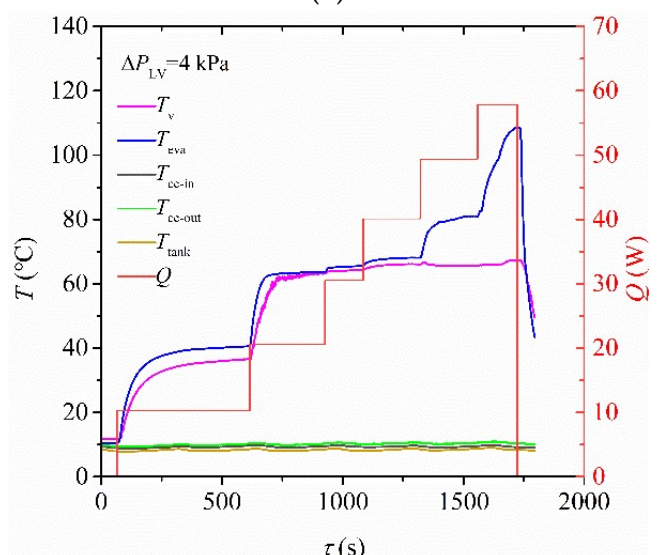

(c)

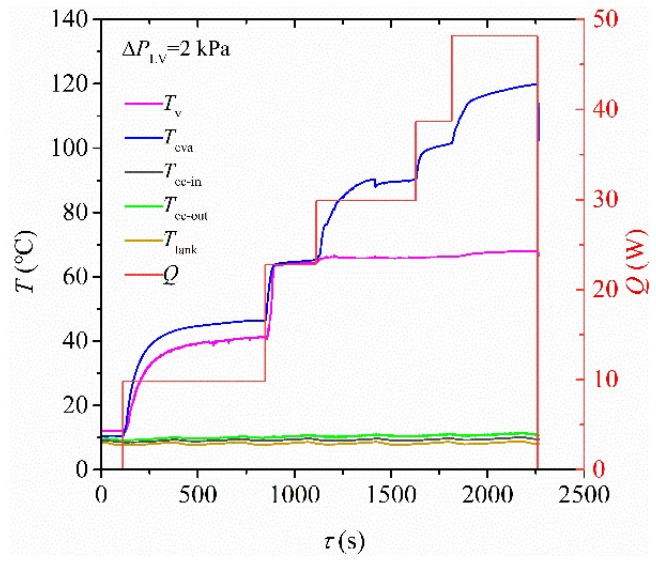

(b)

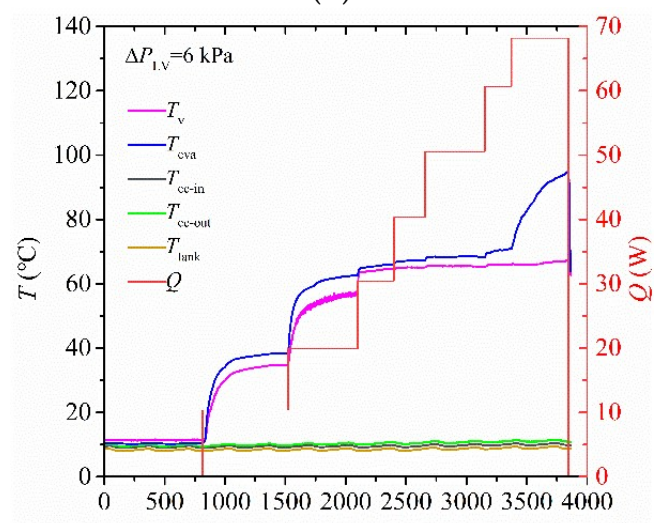

$\tau(\mathrm{s})$

(d) 


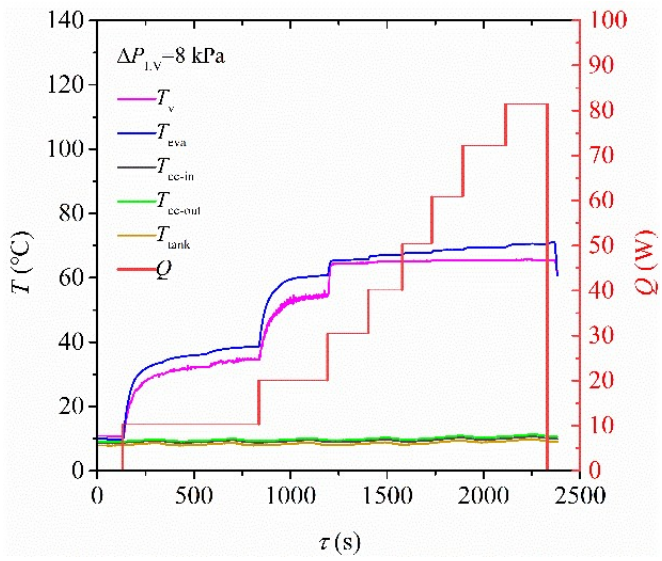

(e)

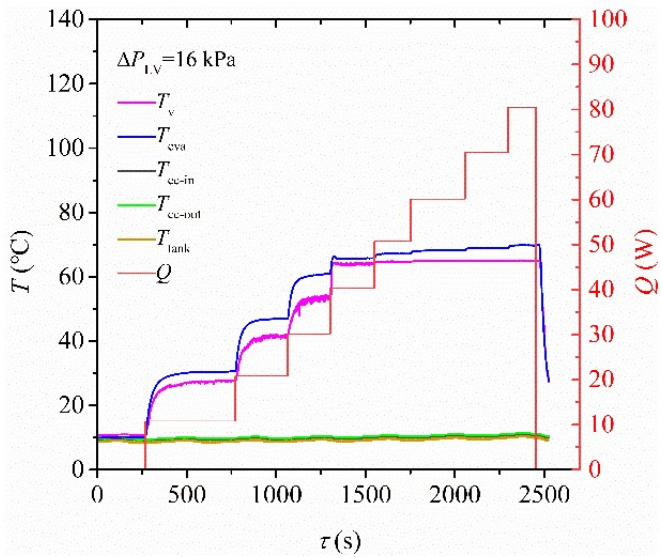

(f)

Figure 3. Start-up characteristics of the hybrid phase-change loop under different pressure differences, (a) $\Delta P_{\mathrm{LV}}=0 \mathrm{kPa}$; (b) $\Delta P_{\mathrm{LV}}=2 \mathrm{kPa}$; (c) $\Delta P_{\mathrm{LV}}=4 \mathrm{kPa}$; (d) $\Delta P_{\mathrm{LV}}=6 \mathrm{kPa}$; (e) $\Delta P_{\mathrm{LV}}=8 \mathrm{kPa}$; (f) $\Delta P_{\mathrm{LV}}=16 \mathrm{kPa}$.

\subsection{Steady state heat transfer characteristics}

The steady-state heat transfer characteristics could directly reflect the heat transfer performance of phase-change loop. This paper presents a systematic study on the steadystate baseplate temperature, heat transfer coefficient and thermal resistance of phasechange loop, with the following two influence factors taken into consideration: liquidvapor pressure difference and heating power.

The heat transfer coefficient of copper baseplate can be calculated according to the following formula

$$
h=\frac{Q}{A\left(T_{\text {eva }}-T_{\mathrm{f}}\right)}
$$

where $Q$ is heating power, $A$ is heat transfer area, $T_{\text {eva }}$ is baseplate temperature, and $T_{\mathrm{f}}$ is fluid temperature at vapor collector outlet.

The thermal resistance of phase-change loop can be calculated according to the following formula

$$
R=\frac{T_{\text {eva }}-T_{\text {sink }}}{Q}
$$

where $T_{\text {sink }}$ is the outlet temperature of the chiller.

\subsubsection{Baseplate temperature}

The variation in the copper baseplate temperature $T_{\text {eva }}$ with the change of liquid-vapor pressure difference and heating power under steady-state conditions are shown in Figure 4. It can be seen that, as long as the liquid-vapor pressure difference was $0 \mathrm{kPa}$ and $2 \mathrm{kPa}$, a small change in heating power may lead to a rapid rise in $T_{\text {eva. }}$. However, when the liquid-vapor pressure difference was $4 \mathrm{kPa}$, there were two obvious turning points in the variation curve of $T_{\text {eva }}$ as the heating power increased. After exceeding the first turning

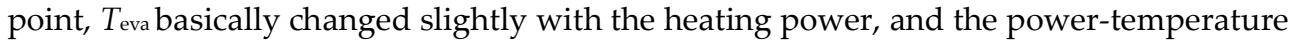
curve became steep. When the heating power continued to increase, a second turning point occurred, and $T$ eva began to increase rapidly again. The first turning point occurred after the heat transfer process was significantly enhanced with the heat transfer mode switching from partial flooded to thin film evaporation. The second turning point occurred after the heat transfer mode switched from thin film evaporation to overheating. When the liquid-vapor pressure difference exceeded $4 \mathrm{kPa}$, the power-temperature curve 
became steep after a turning point. Besides, with the increase of liquid-vapor pressure difference, the heating power at the turning point also increased gradually.

However, high liquid-vapor pressure difference was not always recommended in engineering application. For example, under current experimental conditions, efficient heat dissipation could be achieved by just maintaining liquid-vapor pressure difference lower than $8 \mathrm{kPa}$. But if the liquid-vapor pressure difference exceeded $8 \mathrm{kPa}$, unnecessary pumping power would be caused.

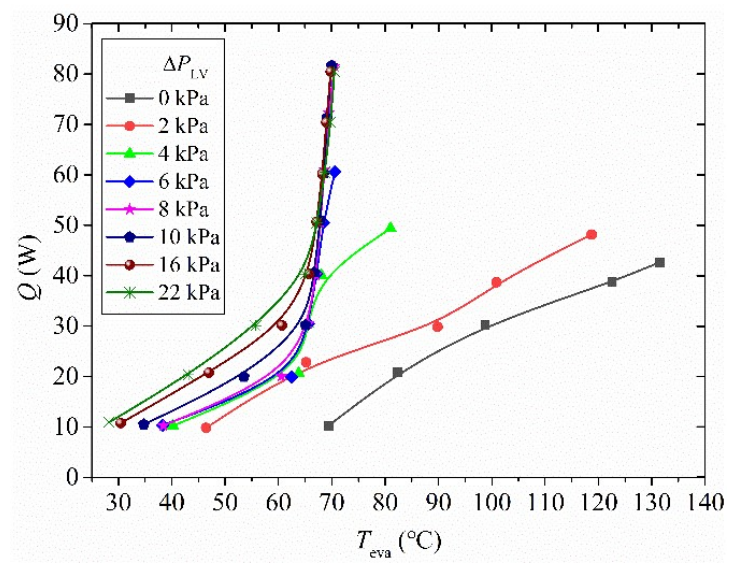

Figure 4. Variation of baseplate temperature under different liquid-vapor pressure difference and heating power.

\subsubsection{Heat transfer characteristics}

The variation of heat transfer coefficient (HTC) of evaporator with the liquid-vapor pressure difference and heating power is shown in Figure 5. Referring to Figure 5, the HTC increases first and then decreased with the increase in heating power under different liquid-vapor pressure differences. Particularly, when the liquid-vapor pressure difference is $0 \mathrm{kPa}$ and $4 \mathrm{kPa}$, the HTC would increase first to the maximum and then decrease rapidly. In contrast, when the liquid-vapor pressure difference exceeded $8 \mathrm{kPa}$, the HTC would decrease gradually after reaching the maximum. Anyway, the change in HTC was closely related to the transition of heat transfer modes. Under low liquid-vapor pressure difference, with the increase of heating power, the heat transfer mode would switch from flooded and partial flooded to thin film evaporation, resulting in rapid increase in HTC. If the heating power continued to increase, the heat transfer mode would switch from thin film evaporation to overheating, leading to a decrease in the HTC. However, under high liquid-vapor pressure difference, there would be no transition from thin film evaporation to overheating at current heating power. Therefore, the HTC would not decrease rapidly.

In summary, when the liquid-vapor pressure difference was low, the range of power required to maintain high HTC was small and it was easy to enter overheating mode. In contrast, high liquid-vapor pressure difference could ensure that high heat transfer coefficient could be maintained in wider power range. Meanwhile, under higher liquid-vapor pressure difference, the HTC would increase rapidly with heating power. During engineering application, the liquid-vapor pressure difference could be actively adjusted according to the operation characteristics of electronic devices in order to achieve the optimum heat dissipation effect. 


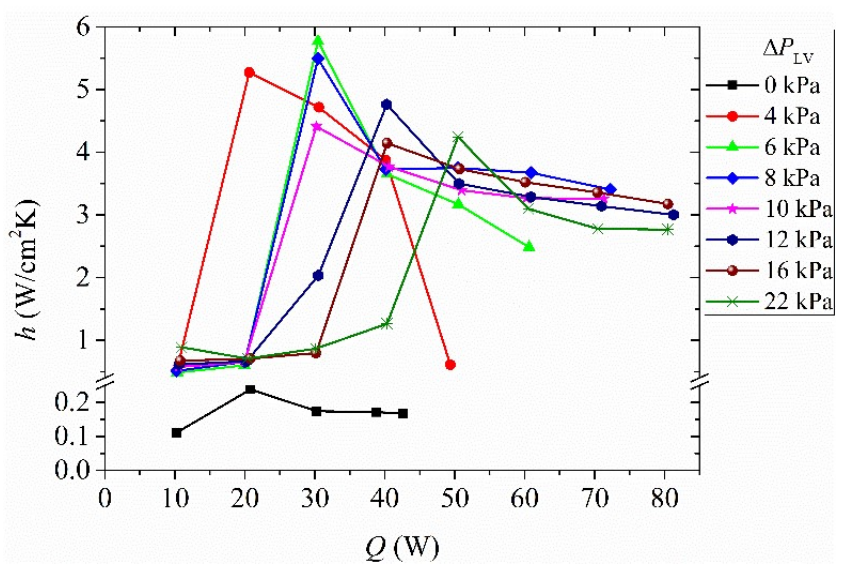

Figure 5. Variation of heat transfer coefficient under different liquid-vapor pressure difference and heating power.

\subsubsection{Regime map of different heat transfer modes}

Figure 6 is the schematic diagram of distribution of vapor phase and liquid phase under different heat transfer modes. The heat transfer modes of baseplate fundamentally depended on the balance between evaporation and liquid replenishment. The evaporation was related to the heating power while the liquid replenishment was related to the driving force (liquid-vapor pressure difference and capillary force). When the liquid replenishment was much greater than the evaporation, the heat transfer process was dominated by convection, leading to flooded or partial flooded modes. However, when the liquid replenishment slightly exceeded the evaporation, a stable evaporating meniscus could be maintained within the vapor channel, the liquid could completely wet the vapor channel, leading to thin film evaporation mode. When the liquid replenishment was slightly lower than the evaporation, the liquid bridge between the liquid and capillary wick in the vapor channel would disappear, and a part of the vapor channel would expose and overheat the generated vapor. At that time, the heat transfer mode was overheating.

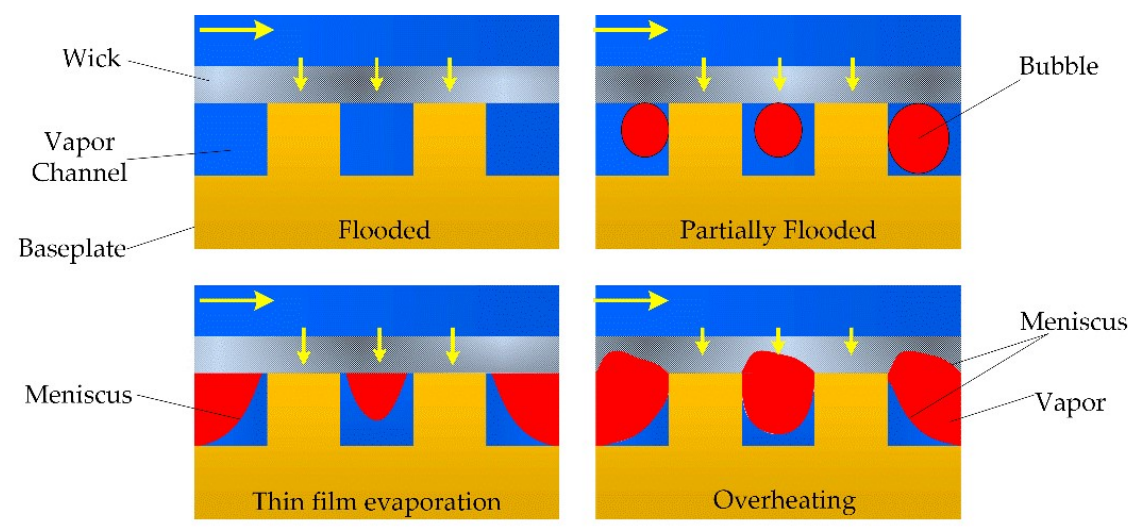

Figure 6. Schematic diagram of four different heat transfer modes.

From the above discussion, thin film evaporation mode was superior to other three heat transfer modes in heat transfer efficiency and start-up speed, and was also the most desirable state in engineering applications. As for copper baseplate, its heat transfer mode was closely related to the liquid-vapor pressure difference and heating power. A twodimensional heat transfer mode distribution diagram considering both liquid-vapor pressure difference and heating power was drawn through an amount of experiments, as shown in Figure 7. Under high liquid-vapor pressure difference or low heating power, the 
liquid replenishment capacity was high, the heat transfer was dominated by single-phase convection, and the heat transfer modes were mainly flooded and partial flooded. Under low liquid-vapor pressure difference or high heating power, the liquid replenishment capacity was low while the evaporation rate was high, the heat transfer mode would switch to overheating. The thin film evaporation mode was between the two, with its distribution area in the shape of a "horn". In engineering application, the liquid-vapor pressure difference could be regulated dynamically according to the heat transfer mode distribution diagram in order to maintain an efficient thin film evaporation mode at a certain heating power.

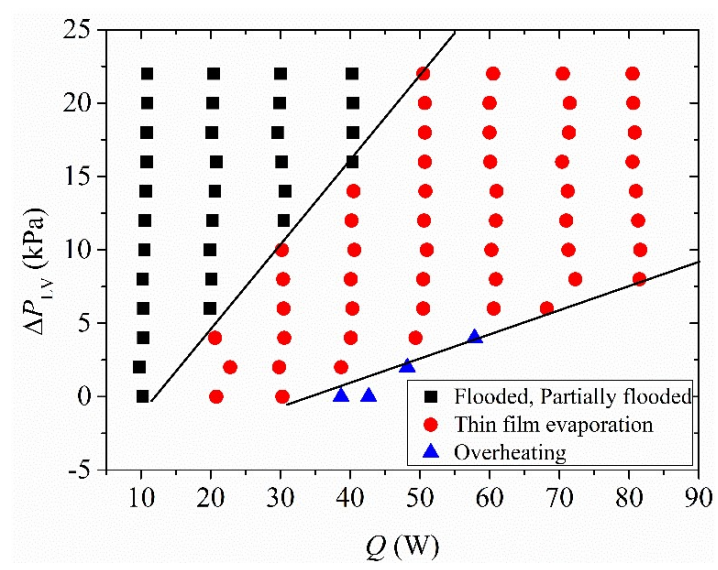

Figure 7. Two-dimensional heat transfer mode distribution diagram.

The variation of thermal resistance of phase-change loop with liquid-vapor pressure difference and heating power is shown in Figure 8. The thermal resistance decreased with the increase of heating power under various pressure differences. When the power is below $40 \mathrm{~W}$, thermal resistance would constantly decrease with the increase of liquid-vapor pressure difference. For example, when the liquid-vapor resistance would decrease from $6.0 \mathrm{~K} / \mathrm{W}$ to $1.8 \mathrm{~K} / \mathrm{W}$, with a decrease of $70 \%$, indicating that heat transfer could be significantly enhanced by increasing liquid-vapor pressure difference at lower power. When the power was higher than $40 \mathrm{~W}$ and the liquid-vapor pressure difference exceeded $8 \mathrm{kPa}$, the thermal resistance curves basically coincided, indicating that the thermal resistance could not be reduced at all times by increasing liquid-vapor pressure difference under high power.

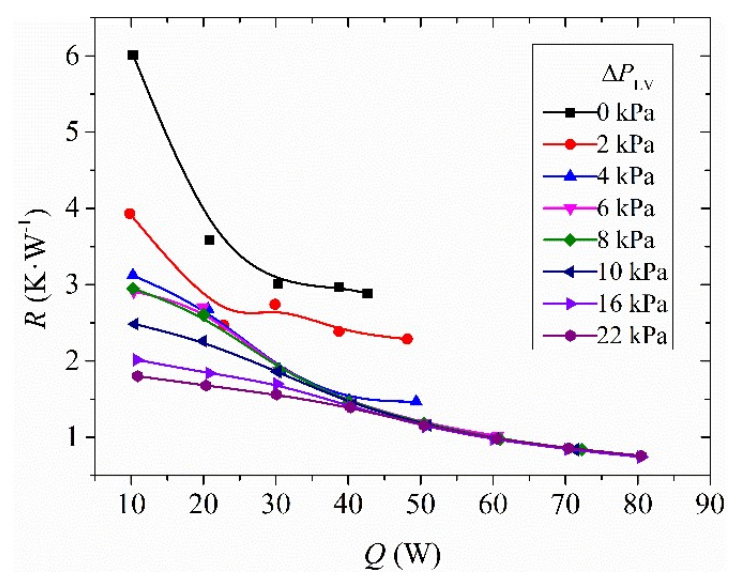

Figure 8. Variation of thermal resistance under different liquid-vapor pressure difference and heating power. 


\section{Conclusions}

In order to solve the problem encountered in the traditional boiling heat dissipation technology i.e. two-phase flow instability, a pump-assisted capillary phase-change loop with methanol as working medium was established. The effect of liquid-vapor pressure difference and heating power on the start-up characteristics and stead-state heat transfer characteristics was studied. The conclusions are as follows

(1) There was no temperature fluctuation in the starting process under various liquidvapor pressure differences and powers, indicating that the pump-assisted capillary phasechange loop overcame the two-phase flow instability. There were four modes of heat transfer including flooded, partial flooded, thin film evaporation and overheating. It took the shortest time (i.e. within 100s) to start up under the thin film evaporation mode. But it took more than 500s under the other three modes.

(2) Both liquid-vapor pressure difference and power were not only important to the heat transfer modes, but also determined the steady-state heat transfer performance of phase-change loop. The heat transfer coefficient can be significantly improved and the thermal resistance can be reduced by increasing liquid-vapor pressure difference as long as it did not exceed $8 \mathrm{kPa}$. For instance, if the liquid-vapor pressure difference increased from $0 \mathrm{kPa}$ to $8 \mathrm{kPa}$ while the heating power remained $30 \mathrm{~W}$, the heat transfer coefficient could be improved by nearly 27 times and the thermal resistance reduced by $37 \%$. When the liquid-vapor pressure difference exceeded $8 \mathrm{kPa}$, the temperature power curve and thermal resistance power curve basically coincided under high heating power. At that time, if the liquid-vapor pressure difference continued to increase, the heat transfer enhancement effect would decrease and more pumping power will be caused.

(3) The thin film evaporation mode with the maximum heat transfer efficiency was the most desirable heat transfer state in engineering applications. A two-dimensional heat transfer mode distribution diagram considering both liquid-vapor pressure difference and heating power was drawn through an amount of experiments. The thin film evaporation mode was between flooded/partial flooded and overheating, in the shape of a "horn". During engineering application, the liquid-vapor pressure difference could be controlled to maintain efficient thin film evaporation at the baseplate of the evaporator in order to achieve the optimum heat dissipation effect.

(4) The capillary wick is a core part of evaporator. Therefore, capillary wick material and its main performance parameters (pore size, porosity and permeability) as well as the type of its combination with the baseplate, which have important effect on the heat transfer performance of phase change loop, deserve in-depth and systematic research in the future.

Author Contributions: Writing-original draft preparation, Xiaoping Yang and Gaoxiang Wang; writing - review and editing, Cancan Zhang; investigation, data curation, Jie Liu; conceptualization, project administration, funding acquisition, Xiaoping Yang and Jinjia Wei. All authors have read and agreed to the published version of the manuscript.

Funding: The research was supported by the NATIONAL NATURAL SCIENCE FOUNDATION OF CHINA, grant number 52006166, 51961135102 and the FUNDAMENTAL RESEARCH FUNDS FOR THE CENTRAL UNIVERSITIES, grant number XJH012020040.

Institutional Review Board Statement: None.

Informed Consent Statement: None.

Acknowledgments: None.

Conflicts of Interest: We declare that we have no financial and personal relationships with other people or organizations that can inappropriately influence our work, there is no conflict of interest in the manuscript entitled "Experimental study on the heat transfer performance of pump-assisted capillary phase-change loop". 
1. Kandlikar S.G. Fundamental issues related to flow boiling in minichannels and microchannels. Experimental Thermal and Fluid Science 2002, 26( 2-4):389-407. (DOI: 10.1016/S0894-1777(02)00150-4)

2. Mudawar, I. Assessment of high-heat-flux thermal management schemes. IEEE Transactions on Components and Packaging Technologies 2001, 24(2):122-141. (DOI: 10.1109/6144.926375)

3. Maydanik Y.F.; Chernysheva M.A.; Pastukhov V.G. Review: Loop heat pipes with flat evaporators. Applied Thermal Engineering 2014, 67(1-2):294-307. (DOI: 10.1016/j.applthermaleng.2014.03.041)

4. Chen P.C.; Lin W.K. The application of capillary pumped loop for cooling of electronic components. Applied Thermal Engineering 2001, 21(17):1739-1754. (DOI: 10.1016/S1359-4311(01)00045-X)

5. Miyazaki Y.; Oshima S.; Furukawa M.; Imai R. Pump-assisted heat pipe system, AIAA Thermophysics. Plasmadynamics and Lasers Conference 1988, Antonio, Texas.

6. Lee J.; Mudawar I. Fluid flow and heat transfer characteristics of low temperature two-phase micro-channel heat sinks - Part 2. Subcooled boiling pressure drop and heat transfer. International Journal of Heat and Mass Transfer 2008, 51(17-18):4327-4341. (DOI: 10.1016/j.ijheatmasstransfer.2008.02.013)

7. An Z.J.; Li J.; Li X.J.; Ding Y. Experimental investigation on lithium-ion battery thermal management based on flow boiling in mini-channel. Applied Thermal Engineering 2017, 117:534-543. (DOI: 10.1016/j.applthermaleng.2017.02.053)

8. Alam T.; Lee P.S.; Yap C.R. Investigation of flow boiling instabilities in silicon microgap heat sink. IEEE 14th Electronics Packaging Technology Conference (EPTC) 2012.

9. Alam T.; Lee P.S.; Yap C.R.; Jin L. A comparative study of flow boiling heat transfer and pressure drop characteristics in microgap and microchannel heat sink and an evaluation of microgap heat sink for hotspot mitigation. International Journal of Heat and Mass Transfer 2013, 58(1-2):335-347. (DOI: 10.1016/j.ijheatmasstransfer.2012.11.020)

10. Ding Y.; Kakac S.; Chen X.J. Dynamic instabilities of boiling two-phase flow in a single horizontal channel. Experimental Thermal and Fluid Science 1995, 11:327-342. (DOI: 10.1016/0894-1777(95)00036-4)

11. Yu Z.T.; Tan S.C.; Yuan H.S.; Cheng C.; Chen X.B. Experimental investigation on flow instability of forced circulation in a minirectangular channel under rolling motion, International Journal of Heat and Mass Transfer 2016, 92, 732-743. (DOI: 10.1016/j.ijheatmasstransfer.2015.09.048)

12. Wu H.Y.; Cheng P. Boiling instability in parallel silicon microchannels at different heat flux. International Journal of Heat and Mass Transfer 2004, 47(17-18), 3631-3641. (DOI: 10.1016/j.ijheatmasstransfer.2004.04.012)

13. Kuang Y.W.; Wang W.; Miao J.Y.; Zhang H.X.; Yu X.G. Non-linear analysis of nitrogen pressure drop instability in micro/minichannels. International Journal of Heat and Mass Transfer 2020, 147, 118953.1-118953.13. (DOI: 10.1016/j.ijheatmasstransfer.2019.118953)

14. Wang G., Cheng P., Wu H.Y. Unstable and stable flow boiling in parallel microchannels and in a single microchannel, International Journal of Heat and Mass Transfer 200750 (21-22), 4297-4310. (DOI: 10.1016/j.ijheatmasstransfer.2007.01.033)

15. Huang H.; Pan L.M.; Yan R.G. Flow characteristics and instability analysis of pressure drop in parallel multiple microchannels, Applied Thermal Engineering 2018, 142, 184-193. (DOI: 10.1016/j.applthermaleng.2018.06.083)

16. Drummond K.P.; Weibel J.A.; Garimella S.V. Experimental study of flow boiling in a compact hierarchical manifold microchannel heat sink array. IEEE Thermal Measurement, Modeling and Management Symposium, 2017, 139-143. (DOI: 10.1109/SEMITHERM.2017.7896921)

17. Drummond K.P.; Back D.; Sinanis M.D.; Janes D.B.; Peroulis D.; Weibel J.A.; Garimella S.V.. Characterization of hierarchical manifold microchannel heat sink arrays under simultaneous background and hotspot heating conditions. International Journal of Heat and Mass Transfer 2018, 126, 1289-1301. (DOI: 10.1016/j.ijheatmasstransfer.2018.05.127)

18. Deng D.X.; Zeng L.; Sun W. A review on flow boiling enhancement and fabrication of enhanced microchannels of microchannel heat sinks. International Journal of Heat and Mass Transfer 2021, 175(2), 121332. (DOI: 10.1016/j.ijheatmasstransfer.2021.121332)

19. Kandlikar S.G. History, advances, and challenges in liquid flow and flow boiling heat transfer in microchannels: a critical review. ASME Journal of Heat Transfer 2012, 134, 034001-1-034001-15. (DOI: 10.1115/IHTC14-23353)

20. Mao N.; Zhuang J.J.; He T.B.; Song M.J. A critical review on measures to suppress flow boiling instabilities in microchannels. Heat and Mass Transfer 2021, 6, 1-22. (DOI: 10.1007/s00231-020-03009-2)

21. Mukherjee A.; Kandlikar S.G. Numerical study of the effect of inlet constriction on flow boiling stability in microchannels. ASME 3rd International Conference on Microchannels and Minichannels 2005, Toronto, Canada.

22. Vutha A.K.; Rao S.R.; Houshmand F.; Peles Y. Active control of flow boiling oscillation amplitude and frequency using a transverse jet in crossflow. Applied Physics Letters 2016, 108, 134104. (DOI: 10.1063/1.4945334)

23. Sourtiji E.; Peles Y. Flow boiling in microchannel with synthetic jet in cross-flow. International Journal of Heat and Mass Transfer 2020 147, 119023. (DOI: 10.1016/j.ijheatmasstransfer.2019.119023) 